\title{
Clinical Trial Registry
}

National Cancer Institute

\section{Source}

National Cancer Institute. Clinical Trial Registry. NCI Thesaurus. Code C142452.

A publicly available database of clinical trial summary information, which may include purpose of the study, recruiting status, condition and medical product under study, study design, trial phase, participation criteria, contact information, primary outcome measure, and results. 